DOI: https://doi.org/10.47405/mjssh.v6i1.617

\begin{tabular}{|c|c|}
\hline 8 & Malaysian Journal of Social Sciences and Humanities (MJSSH) \\
\hline Malaysian Journal of & Volume 6, Issue 1, January 2021 \\
\hline $\begin{array}{l}\text { Humantives } \\
\text { (MJ-SSH) }\end{array}$ & e-ISSN : 2504-8562 \\
\hline & $\begin{array}{l}\text { Journal home page: } \\
\text { www.msocialsciences.com }\end{array}$ \\
\hline
\end{tabular}

\title{
Disclosure of Herbal Medicine Usage in Diabetes Management: A Qualitative Study amongst Type 2 Diabetes Mellitus Patients and Health Care Providers in Negeri Sembilan, Malaysia
}

\author{
Nadia Amirudin'1, Albeny Joslyn Panting1 , Rosnani Kassim¹, Norbaidurah Ithnain'1, \\ Manimaran Krishnan' \\ ${ }^{1}$ Institute for Health Behavioural Research, Ministry of Health Malaysia \\ Correspondence: Nadia Amirudin (nadia.amr@moh.gov.my)
}

\begin{abstract}
The use of herbal medicine (HM) and its products has significantly increased in the past decade. Type 2 Diabetes mellitus patients (T2DM) are always rendering herbal medicine as an alternative to the current option of treatment to manage their disease. This article is aimed to explore the extent of communication process of patients who disclose their HM usage to healthcare providers (HCPs) and HCP's experiences in managing these patients. Purposive sampling method was applied in this qualitative study, which involved 28 respondents from four government clinics in Negeri Sembilan, Malaysia. A semi-structured interview was developed for the in-depth interview (IDI) and focus group interview (FGD). Both IDIs and FGDs were audio-recorded and conducted for approximately (40-60) minutes. The contents were transcribed verbatim and thematically analysed. The majority of T2DM patients did not reveal their HM usage due to fear of negative feedback, never been inquired by HCP and bad experiences after disclosure. Whereas, patients will disclose if they have a good rapport with HCPs and HCP asked patients courteously. However, HCPs reported that they did not discuss HM usage with patients due to limited knowledge, patients refuse to admit and time constraint to consult patients. Communication between T2DM patients and HCPs during consultation concerning the proper use of HM alongside modern medicine needs to be improved. Therefore, a better understanding factor of HM usage and its disclosure would make patients more open-minded to discuss and encourage HCPs to be attentive to avoid any potential adverse effects in HM usage.
\end{abstract}

Keywords: herbal medicine, T2DM, disclosure, non-disclosure, experience, communication

\section{Introduction}

A global report on Herbal Supplements and Remedies market shows that the demand for HM in healthcare practice continues to grow worldwide and by 2017, the global market for such products is expected to be US $\$ 104.6$ billion. Studies reported that among the most preferred Complementary and Alternative medicine (CAM) therapies pursued by chronic patients like diabetics are herbal remedies (Boston et al., 2019; Pearson et al., 2018; Jiaranaikajorn et al., 2017; Peltzer et al., 2016). Herbal Medicines (HM) have been acknowledged as part of traditional medicines which include herbal plants, herbal ingredients and finished herbal products that contain plant parts as active components. Even though the HM has been widely used and carries potential risks, most studies revealed that HM usage is still hardly discussed between patients with their doctors. 
According to Liem A. (2019), the national survey conducted by Indonesian Health Ministry reported more than $75 \%$ of Indonesian people using CAM treatments especially herbal medicines. However, the majority of them might not disclose their usage to HCP and presumed it should not be discussed if they find their HCP is being skeptical. Another study done by Kretchy et al. (2014) in Ghana reported the main reasons of non-disclosure by $70 \%$ respondent of CAM user is due to fear of the doctor and lack of inquiry by HCP. Similarly, negative attitudes and belief towards CAM by health practitioners make patients reluctant to openly discuss with them. A study in Australia found that there is a correlation between knowledge and attitude that is most likely to be negative for health practitioners with limited knowledge of CAM (Shorofi \& Arbon 2017).

In addition, Toklu (2016) claimed that HM usage is always associated with a few problems, which are herb-drug interaction, improper labelling, contamination, unknown composition, lack of standardisation for active components dosage and inappropriate dose. Although herbal products are thought to be harmless amongst patients, there may be side effects and potentials to cause drug interactions (Tuluney et al. 2015); hence, the study stated that adverse drug effects, toxicity, and treatment failure were more likely to occur when herbal products were consumed with conventional medicines. Moreover, with intensive usage but limited disclosure of HM, patients may expose themselves to risks, for instance, making uninformed decisions regarding other treatments, involving drug interactions, misdiagnosis and mismanagement of their diseases (Liem, A., 2019). As a consequence, serious complications can occur and affect the healing period of their illness.

The growing use of herbs requires appropriate rules and regulations under the Medical Act for safety reasons. HCPs should be aware of their patient's HM usage and advise them of the potential unwanted side effects in medicine-drug interaction. A better understanding of HM usage will help patients to be more open-minded to discuss with HCPs and allow HCP to be attentive and patient-centred, particularly during counselling sessions with regard to proper usage of herbal remedies. Therefore, this study was intended to explore the communication process and determine the extent that patients disclose their HM usage to HCPs and HCP's experiences in managing the patient's HM usage.

\section{Methods}

\section{Study Design}

The study sample consisted of T2DM patients and HCPs selected from four government clinics in Negeri Sembilan, namely Seremban Health Clinic, Port Dickson Health Clinic, Kuala Pilah Health Clinic, and Bahau Health Clinic. Selection of clinics was based on the highest number of diabetes patients registered at the clinics.

\section{Data Collection}

For interview process, the researcher used primarily open-ended questions to extract full information from the informants and applied the probing techniques. The interview took 40-60 minutes for the interviewer to complete, while a research assistant was tasked with taking notes. However, informants were assembled as a group for the FGD session. The discussion was driven by a semi-structured questionnaire consisting of questions on the use of herbal medicine and their patient management experience. One researcher acted as a moderator and the other research assistant as a note taker. Thematic saturation has been reached when the researcher found no more new themes emerged from the data.

Both IDIs and FGDs were conducted in a closed room. Samples were selected via purposive sampling and according to their eligibility, which included: Malaysian who were between 18 years and above, diagnosed with diabetes for more than three years, and used HM in managing diabetes for 12 months and above. However, the participating HCP group in this study was selected from medical staff working in the Out Patients Department. An appointment for IDI and FGD sessions was scheduled when the participation was confirmed. 


\section{Data Analysis}

Both IDIs and FGDs were audio-recorded and the contents were transcribed verbatim. However, field notes were taken by research assistant to interpret the data especially for non-verbal communication. The data were thematically analysed and were sorted into various themes and subthemes. The analysis started with listening to the audio-recording and reading each transcript line by line to identify and extract data to initial codes relevant to the study objectives. Then, similar codes were applied to all transcripts and the themes or subthemes were identified. The various themes were identified from literature. New themes also arose spontaneously from informants during the interviews. The thematic analysis was done after peer checking and before the final analysis was conducted. All the data were managed manually by using inductive coding in Excel.

\section{Ethical Consideration}

Ethical approval was obtained from the Medical Research Ethics Committee (MREC), Ministry of Health with an approval number (NMRR ID: 17-953-35849), whereas the approval to conduct this study was granted at the state level and heads of departments from respective clinics. Written consent was obtained from respondents prior to the audio-recording interviews for data collection and they were reassured of their confidentiality and anonymity. In addition, this study was funded by NIH grant.

\section{Results}

\section{Summary of Informants}

A total of 47 respondents were recruited for this study, and they were aged from 30 to 60 years. The multiracial groups had 28 patients, which comprised three main ethnicities in Malaysia: Malays, Chinese and Indians. All patients were diagnosed with DM and have on-going treatment with respective government health clinics in Negeri Sembilan for more than three years. However, 19 respondents who were recruited from the HCP group consisted of Diabetes Nurse Educator, Medical Officer, Family Medicine Specialist, Staff Nurse and Medical Assistant.

\section{Factors of Non-disclosure of HM Usage}

Respondents were asked to describe potential factors that enabled them to disclose their HM usage as the primary choice of treatment for diabetes. Based on the study findings, the most prompted reason was fear of negative feedback from HCPs. The majority of patients informed that they feared if the HCPs will scold them and believed that the HCPs will also disapprove their self-practice of HM usage. Besides, patients reported that the HCPs never inquired them regarding their HM usage during consultation. Therefore, they were not really keen to disclose. They prefer to keep it as a secret until they were asked personally. However, some patients revealed that they did tell the HCPs about HM usage but were reluctant to share it anymore because they had bad experience after disclosure.

\section{Fear of negative feedback}

Half of the patients never thought of disclosing their HM usage to HCPs because they fear of getting negative feedback from them. Patients were afraid of being scolded if they informed about their HM usage to HCPs and perceived that most HCPs did not believe in HM, and thus they will be rejected. Therefore, most patients presumed that it was unnecessary to share about the HM usage, unless the HCP asked them nicely.

"But later if I asked the doctor, I was afraid if the doctor would be angry, take this medicine, but I still want to take traditional meds too. That's why I'm afraid to ask, later I get scolded" (PT6, Female, 66, Chinese) 
"No lah, I'm afraid...If I tell him, he will scold me. If they ask me to fill up a form or ask me if I have taking any herbal meds, I will say no". (PT17, Female, 51, Indian)

"In my opinion, mostly doctors they didn't believe in traditional meds, that's why I didn't tell..." (PT23, Male, 69, Malay)

\section{Never been inquired by HCP}

In some cases, patients did not reveal their HM usage because most of the time HCP never questioned them, and as usual it was not part of the patient-HCP discussion. Besides, a patient once reported that the HCP will provide conventional medicines only as a routine during consultation, together with common diabetes management advice.

"Because the doctor never asks me, he just add medicine, and advise me to control eating, that's it lah. Never think of it (to tell doctor) because doctor never ask, I also never think about it..."(PT3, Female, 66, Indian)

"No, if the doctor asks me, maybe I will tell him. Last time the nurse did ask me (about taking herbs), if she didn't ask, I won't tell her also..."(PT23, Male, 69, Malay)

"Not yet. Never. They never ask, so I keep silent lah" (laughing). (PT12, Male, 64, Malay)

\section{Bad experiences after disclosure}

Patients reported they encountered unpleasant experiences when they informed about their HM usage. Once a patient was scolded and the HCP was being sarcastic about complementing both medicines; hence, patient feels demotivated to reveal their HM usage again in the future.

"I don't like any doctor. I got scolded. I always getting scold by them". (PT17, Female, 51, Indian)

"Err, I told one of private doctors once, I said. Normally, I take that Murungai leaves, traditional thing, he said if you took traditional herbs then why you come for this western medicine? He asked me like that. So, starting from that time, I didn't tell the doctor anything". (PT2, Female, 63, Indian)

\section{Factors of Disclosure of Herbal Medicine Usage}

Despite the bad experience, several informants voluntarily disclosed their herbal intake due to a good relationship with the HCPs. They want to get professional advice and usually, the HCPs will give positive feedback by encouraging them to proceed together with $\mathrm{CM}$ if their HbAlc result was good. Besides, some patients informed that they disclosed when the HCPs initiated a conversation and enquired nicely regarding the HM usage. Therefore, it is crucial for HCPs to acknowledge HM usage by learning how to discuss it with their patients. The HCPs should be "open-minded" so that in the future patients will be more confident to share.

\section{Patients having a good rapport with HCP}

HCPs mentioned that some patients openly informed about their HM usage due to a good relationship with the HCPs and they need professional advice on this matter. Similarly, some patients stated that 
they felt comfortable and received positive feedback (advice), especially if their HbA1c result was good. However, the HCP would also advise them not to forget to take CM.

"...Sometimes when patients arrived at the clinic, if she closes with us, she will story, Haaa... I took this and that, and then we can ask more. If they are close with us, they will tell stories". (HCP 15, Female, 49, Malay)

"Indeed yes, there are patients who consume HM and admit to me and their HbAlc result is very good. For example, I got one Indian patient who confessed to me she took ginger every day. She blends that and boiled it from one cup until becomes the half cup, then she drinks it every morning without a miss. Aaa...she used to take Metformin 850mg BD before, but now only $250 \mathrm{mg}$ OD... and her HbAlc never more than 6.2". (HCP 2, Female, 34, Malay)

“...No lah, but mostly who admit it will ask me, doctor, is this medicine safe to consume?". (HCP 10, Male, 32, Malay)

"I show them lah but they said if you want to take okay no problem lah but make sure you take your medication. They said you don't forget to take your medicine". (PT25, Male, 61, Indian)

\section{HCP asked patients courteously}

Nevertheless, there were cases, whereby the HCPs need to initiate a better approach to make the patients disclose their HM usage. Usually, HCPs will ask when there are changes in the diabetes condition. As modern medical practitioners, HCPs justified that even if they know patients are taking $\mathrm{HM}$, they are prohibited from suggesting any other alternative medicines. Therefore, they start a conversation by sharing a story and asked patients courteously to share their HM usage.

"For example, I will ask ermm...have you take any other meds? If she said no, I will tell her, it's okey. If you have taken, just tell me...I won't scold you, I'm just asking, if you have taken any, I would like to know. Aaaa, if I told like that, then she will voluntarily admit it". (HCP 3, Female, 35, Malay)

"Usually, if before this I notice any changes and become worse, I will ask them, and they will story to me what exactly happened". (HCP 13, Female, 49, Malay)

"I used to share a story, like me... honestly, ermm, from my experience ermm... we are not allowed actually, at the first place to propose or suggest any alternative medicine even though we know it works for certain people. But the way we ask is, I just want to share a story, it's okey...you can come and show me. I'll try to tell you to explain what it is". (HCP 1, Male, 38, Indian)

\section{HCP's experiences in dealing with patients}

Most HCPs stated that they could not give proper advice as they did not have sufficient knowledge about HM, which hindered them from encouraging patients on the usage. They claimed that the limitation made patients refuse to admit, and thus, there was no need to discuss further on that matter. In general, the majority of HCPs confessed that they did not have enough time to consult patients who wanted to disclose their HM usage. Therefore, it was observed that the distinct attitudes between patients and HCPs will decrease the chances for patients to disclose their HM usage to HCPs. 
DOI: https://doi.org/10.47405/mjssh.v6i1.617

\section{Unable to advise patient due to limited knowledge}

HCPs reported they were unable to give proper advice to patients, except to ask them to stop because they know that their knowledge about HM was limited and beyond their field. Moreover, they are not well-trained to give any advice and perceived that HM lacks scientific evidence of efficacy. Even though they admitted they were worried, but they never asked about HM usage in diabetes selfmanagement. "Honestly, when I see patient, I seldom ask them about herbal medicine. And if I
did ask, I won't go into detail because we don't know what to ask and we know
nothing about that herbs. So, usually I will advise them to stop taking HM, but take
conventional meds only because herbs have no research or evidence based on their
efficacy or any side effects. What makes me worry is kidney failure, liver damage
could happen to them. Aaa, but I never ask them further about consuming that
(HM)". (HCP 9, Male, 28, Malay)

“As we know, our knowledge about HM truly limited and we don't know what are the ingredients. Sometimes they bought from outside, they put something harmful like steroid and so on. So, it will be more dangerous. I think I have been quite strict about that and tell them to stop immediately". (HCP 11, Female, 30, Malay)

"Because you have patient who just come and then they ask us what is this, is it safe to take? But there are 100 billion things out there you know and we are not trained to, to actually give them some advice".(HCP 1, Male, 38, Indian)

"I will say, okey I don't have any knowledge on that part to say it is wrong...". (HCP 7, Female, 36, Malay)

\section{Patients refuse to admit}

Majority of HCPs commented that most patients will deny or lie about HM usage after being asked because patients perceived HCPs were being sceptical and not interested. Patients were worried and aware they were at risk to get adverse effects.

"No...Most of them will deny". (HCP 4, Female, 48, Indian)

"Aaa so sometimes yes. Patients in denial it's just that ermm...the way we ask must capture with what they are taking actually". (HCP 3, Female, 35, Malay)

"Haa...some people they think conventional meds is harmful. That's why they want to try herbal medicine. But when we asked... they still don't want to admit lah...". (HCP 9, Male, 28, Malay)

\section{Time constraint to consult patients}

HCPs claimed that time constraint is the main reason why they did not consult patients in detail regarding the HM usage. The reason for HCPs could not commit further discussion on HM usage was a greater number of patients waiting for consultation.

"So, alternative medication is actually very time consuming for me personally because of the restrain in time, it's very hard to go in-depth with that. Ermm... a lot of patients do talk to me but I can't go much in it'.'(HCP 5, Female, 42, Indian).

"The sharing of taking alternative medication for me personally is very limited because of time factor. You want to talk more but you know at the same time you need to key-in all everything". (HCP 4, Female, 48, Indian) 
"Honestly, I'm hardly ask my patient whether they have taken traditional medication or not, and usually when we see so many patients, we want to be fast (finish to consult for all patients)". (HCP 10, Male, 32, Malay)

\section{Discussion}

The present study demonstrated the communication process between patients and HCPs to comprehend to which extent patients' disclosure about the use of HM to HCPs and HCP's experiences in treating diabetes patients using HM. The majority of patients showed poor communication towards disclosing their HM usage to their HCPs due to fear of negative feedback. This was explained by several studies which mentioned the fear of doctor-rejection and disagreement on CAM usage as the major reason of non-disclosure (Ibrahim et al., 2018; Jou \& Johnson 2016; Hwang et al., 2015; Kretchy et al., 2014). Moreover, this was consistent with a study done by Liem A. (2019), which cited patients unlikely to disclose their self-care practices including CAM use because they concern about doctor's feedback. Similarly, HCP's negative or judgmental remarks made patients reluctant to share their HM usage (Mekuria et al., 2018 and Avila et al., 2020).

In addition, patients in this study underlined the manner in which HCP never asked them regarding HM usage during the consultation. HCPs will mostly continue to prescribe conventional medicine and giving some advice regarding diabetes management without asking further unless if they noticed extraordinary results in $\mathrm{HbA} 1 \mathrm{c}$. A similar finding was reported by a previous study conducted by Jou \& Johnson (2016) in the United States which found the most cited reason for non-disclosure was the doctor did not inquire about the use of CAM. It is uncommon to discuss with a patient about CAM unless the patient initiates the conversation (Shelley et al., 2009). Another study also reported that almost all physicians did not ask patients about their HM usage history (Memon et al., 2017; Zhang et al., 2012). Meanwhile, Ibrahim et al. (2018) also quoted comparable findings in which all respondents reported their doctors never asked regarding any treatment with CAM and very few of them disclose their CAM usage to the doctors. Therefore, it is not surprising healthcare professionals are not always aware of CAM used by patients.

On the other hand, several informants in the current study revealed that they had a bad personal experience after they confessed about their HM usage because the HCPs scolded them and were being sarcastic. It made them feel sad, scared and demotivated simultaneously. This might explain why patients refuse to disclose the HM usage alongside taking their conventional medicine. In addition, this finding is comparable to other study which reported that their respondent received a negative reaction from the healthcare professional upon disclosure (Liem A. 2019; Jou \& Johnson 2016). A negative or passive response should be avoided by HCPs while treating the patient as it may result in a decreased patient's health status and bear a higher cost in the healthcare system due to mistreatment to the patient (Chang et al., 2011).

Meanwhile, another key finding obtained from this study was that patients disclose their HM usage due to good relation with their HCPs and feel comfortable to share. Developing virtuous relationships will make patients feel secure to share further details about their health and care expectations (Hamed Abdalla et al., 2020). Patients usually will receive positive feedbacks, especially if they get good $\mathrm{HbA1c}$ results. This finding was in line with another study which found that patients admitted their use of HM after observed their HbA1c results was improved (Memon et al., 2017). Moreover, this study revealed that patients disclose to their HCPs because they want to get professional advice with hope HCPs will support them to continue taking HM. Most HCPs who have a positive attitude towards HM will allow them to take HM as complementary to CM. They believed that the efficacy of HM will help patients to manage their diabetes better than before.

Besides, the present study revealed that a few patients disclosed their HM usage, especially after being politely asked or persuaded. The HCP's attitude towards HM also affected the patient's willingness to openly disclose their HM usage. This was parallel with a study done by Bello et al. (2012) which indicated patients were willing to answer questions and disclose when they were concerned about 
CAM adverse side effects, HCPs showed good attitudes towards them and asked in a good manner. A friendly approach by HCPs could help patients to boost their confidence in revealing their HM usage and make it easier for HCPs to share advice on potential adverse effects of HM such as palpitations, abdominal pain and hot flushing after taking HM for diabetes treatment (Soner et al., 2013).

In regard to HCP's experience in dealing with diabetic patients, the majority of HCPs in this study claimed they were unable to advise patients properly unless they asked the patients to stop taking HM due to inadequate of knowledge HM and it was beyond their field. Moreover, it was difficult to make an informed judgment for the patients as it could contradict the evidence-based principle of the medical stream which can affect their professionalism. This is consistent with a study done by Babar et al. (2012) who reported similar findings where HCP who lacking of CAM knowledge are unable to counsel patients on CAM usage. Memon et al. (2017) and Zhang et al. (2012) also indicate that patients did not discuss CAM with their doctor because they perceived the doctor was ignorant and having insufficient knowledge. This finding clearly showed that doctor needs to be knowledgeable as it is essential to be a well-rounded professional to properly advise patients.

Nonetheless, HCPs commented that most patients would deny even though sometimes they did ask their patients regarding HM usage. Meanwhile, this study revealed that patients will not initiate the topic in the discussion if they notice HCPs are being skeptical and not interested. Besides, the correlation analysis of Ibrahim et al. (2018) study revealed that an inverse relation which patients who have demonstrated a favourable attitude towards CAM were found to rarely disclose to their HCPs. This may imply on the risk of inaccurate diagnosis and serious adverse effects. An open discussion should be held to avoid miscommunication between patients and HCPs. Moreover, patients believed that it was unnecessary to ask HCPs as they may not have good knowledge about HM usage. A study by Arcury et al. (2012) and Hasan et al. (2009) also reported the same findings, whereby the majority of CAM users denied the necessity of asking doctors and did not accept that doctors have good CAMrelated knowledge.

Furthermore, this study highlighted on HCPs never assessed the use of HM due to time constraints. HM usage was rarely discussed by both groups of patients and HCPs. This finding was similar to a study done by Kamel et al. (2017), whereby the majority of patients reported that they never discussed their HM utilisation with their physicians. Another study done by Ibrahim et al. (2018) also reported the same findings, in which the majority of respondents $(82.8 \%)$ agreed that doctors did not have enough time to discuss CAM. Similarly, another consensus was found in several studies in which most patients agreed that insufficient consultation time with their HCP was a barrier to disclose their CAM usage and therefore, they never thought to bring up for discussion (Jou \& Johnson 2016; Chiba et al., 2014; Zhang et al., 2012).

\section{Conclusion}

In conclusion, this study has identified patient's concerns towards fear of negative feedback, never been inquired by HCP and bad experience after disclosure as a major reasons of HM non-disclosure. On the other hand, patients decided to disclose their use of HM due to a good rapport with HCP and were courteously persuaded. These findings might help HCP to improve their communication skills and practice more during consultation time. Besides that, understanding patient usage for both types of medicine may be valuable to minimize the risks of HM-CM potential drug interaction. Therefore, patient's behaviour and communication skills concerning HM consumption should be highlighted through the health education programme. In a nutshell, understanding views from both groups is crucial to develop an opportunity for patients to have a better self-care in diabetes management and for $\mathrm{HCP}$ to deliver effective patient care. 


\section{Acknowledgment}

We would like to thank the Director-General of Health and Deputy Director-General of Health (Research and Technical Support), Ministry of Health Malaysia for the permission to publish this paper.

\section{Competing Interests Statement}

The authors declare that there are no competing or potential conflicts of interest.

\section{References}

Adofo, S. (2013). Challenges And Coping Strategies Of Student Nursing Mothers In Tertiary Institutions In The Greater Accra Region Of Ghana (M.Phil). University of Ghana.

Arcury, T. A., Bell, R. A., Altizer, K. P., Grzywacz, J. G., Sandberg, J. C., \& Quandt, S. A. (2012). Attitudes of Older Adults Regarding Disclosure of Complementary Therapy Use to Physicians. Journal of Applied Gerontology, 32(5), 627 645. https://DOI.ORG/10.1177/0733464812443084

Avila, C., Grace, S., \& Bradbury, J. (2020). How do patients integrate complementary medicine with mainstream healthcare? A survey of patients' perspectives. Complementary Therapies in Medicine, 49(January), 102317. https://doi.org/10.1016/j.ctim.2020.102317

Babar, M. G., Syed, S. H., Naing, C. M., \& Hamzah, N. H. B. (2012). Perceptions and self-use of Complementary and Alternative Medicine (CAM) among Malaysian dental students. European Journal of Integrative Medicine, 4(1). https://doi.org/ 10.1016/j.eujim.2011.11.001

Bello, N., Winit-Watjana, W., Baquir, W., \& McGarry, K. (2012). Disclosure and adverse effects of complementary and alternative medicine used by hospitalized patients in the North East of England. Pharmacy Practice (Internet), 10(3), 125-135.

Boston, C., Wong, N., Ganga, T., Chandradatt, K., Rosales, J., Singh, J., \& Kurup, R. (2019). Comparison and Effectiveness of Complementary and Alternative Medicine as against Conventional Medicine in the Treatment and Management of Type 2 Diabetes. Journal of Complementary and Alternative Medical Research, 7(2), 1-8. https://doi.org/10.9734/jocamr/2019/v7i230097

Chang, H. Y. A., Wallis, M., \& Tiralongo, E. (2011). Use of complementary and alternative medicine among people with type 2 diabetes in Taiwan: A cross-sectional survey. Evidence-Based Complementary and Alternative Medicine, 2011. https://doi.org/10.1155/2011/983792

Global Herbal Supplements and Remedies Market to Reach US \$ 107 Billion.

(2012 March 07). Retrieved from http://www.prweb.com/releases/herbal_supplements/herbal_remedies/prweb9260421.htm

Hamed Abdalla, M. E. A., Ali, A. M., \& Loong, L (2020). The use of complementary and alternative medicine (CAM) among cancer patients at a tertiary hospital in Malaysia. Complementary Therapies in Medicine, 50, 102343. https://doi.org/10.1016/j.ctim.2020.102343

Hasan, S. S., Ahmed, S. I., Bukhari, N. I., \& Loon, W. C. W. (2009). Use of complementary and alternative medicine among patients with chronic diseases at outpatient clinics. Complementary Therapies in Clinical Practice, 15(3), 152-157. https://doi.org/10.1016/j.ctcp.2009.02.003

Hwang, J. H., Kim, W.-Y., Ahmed, M., Choi, S., Kim, J., \& Han, D. W. (2015). The Use of Complementary and Alternative Medicine by Korean Breast Cancer Women: Is It Associated with Severity of Symptoms? Evidence-Based Complementary and Alternative Medicine, 2015, 1-7. https://doi.org/10.1155/2015/182475

Ibrahim, I. R., Hassali, M. A., Saleem F., Tukmagi, H. F., \& Dawood, O. T. (2018). Patients' Attitudes towards Complementary and Alternative Medicine: A Cross-Sectional Study among Hypertensive Patients in Baghdad, Iraq. Journal of Complementary Medicine \& Alternative Healthcare, 7(3). https://doi.org/10.19080/jcmah.2018.07.555713

Jiaranaikajorn, T., Panthawangkul, J., \& Thamlikitkul, V. (2002). Use of Alternative Medicines among Medical Patients at Siriraj Hospital. Siriraj Medical Journal, 54(10), 603-610. Retrieved from 
DOI: https://doi.org/10.47405/mjssh.v6i1.617

https://he02.tci-thaijo.org/index.php/sirirajmedj/article/view/245316

Jou, J., \& Johnson, P. J. (2016). Nondisclosure of Complementary and Alternative Medicine Use to Primary Care Physicians. JAMA Internal Medicine, 176 (4), 545. https://doi.org/10.1001/jamainternmed.2015.8593

Kamel, F. O., Magadmi, R. M., Hagras, M. M., Magadmi, B., \& AlAhmad, R. A. (2017). Knowledge, attitude, and beliefs toward traditional herbal medicine use among diabetics in Jeddah Saudi Arabia. Complementary Therapies in Clinical Practice, 29, 207-212. https://doi.org/10.1016/j.ctcp.2017.10.007

Kretchy, I. A., Owusu-Daaku, F., \& Danquah, S. (2014). Patterns and determinants of the use of complementary and alternative medicine: a cross-sectional study of hypertensive patients in Ghana. BMC Complementary and Alternative Medicine, 14(1). https://doi.org/10.1186/14726882-14-44

Liem, Andrian (2019). Beliefs, attitudes towards, and experiences of using complementary and alternative medicine: A qualitative study of clinical psychologists in Indonesia. European Journal of Integrative Medicine, 26( ), 1-10. https://doi.org/10.1016/j.eujim.2019.01.003

Mekuria, A. B., Belachew, S. A., Tegegn, H. G., Ali, D. S., Netere, A. K., Lemlemu, E., \& Erku, D. A. (2018). Prevalence and correlates of herbal medicine use among type 2 diabetic patients in Teaching Hospital in Ethiopia: A cross-sectional study. BMC Complementary and Alternative Medicine, 18(1), 1-8. https://doi.org/10.1186/s12906-018-2147-3

Memon, A. R., Randhawa, M. A., Arain, A. A., Alrawaili, Y. S. H., K, Y. S., Alrawaili, N. F. A., Alhassan, N. S., Alshammari, S. O. M., \& Alanazi, A. M. S. (2017). Herbal Medicine Use: Knowledge and Attitude in Patients At Tertiary Care Level in Northern Border Region of Kingdom of Saudi Arabia. Journal of Sheikh Zayed Medical College (JSZMC);8(3): 1241-1244.

Pearson, H., Fleming, T., Chhoun, P., Tuot, S., Brody, C., \& Yi, S. (2018). Prevalence of and factors associated with utilization of herbal medicines among outpatients in primary health centers in Cambodia. BMC Complementary and Alternative Medicine, 18(1). https://doi.org/10.1186/s12906-018-2181-1

Peltzer K, Pengpid S, Puckpinyo A, Yi S, Anh Le V. The utilization of traditional, complementary and alternative medicine for non-communicable diseases and mental disorders in health care patients in Cambodia, Thailand and Vietnam. BMC Complement Altern Med. (2016);16:92. https://doi.org/10.1186/s12906-016-1078-0.

Shelley, B. M.; Sussman, A. L.; Williams, R. L.; Segal, A. R.; Crabtree, B. F. (2009). 'They Don't Ask Me So I Don't Tell Them': Patient-Clinician Communication About Traditional, Complementary, and Alternative Medicine. The Annals of Family Medicine, 7(2), 139147.https://doi.org/10.1370/afm.947

Shorofi, S. A., \& Arbon, P. (2017). Complementary and alternative medicine (CAM) among Australian hospital-based nurses: knowledge, attitude, personal and professional use, reasons for use, CAM referrals, and socio-demographic predictors of CAM users. Complementary Therapies in Clinical Practice, 27, 37-45. https://doi.org/10.1016/j.ctcp.2017.03.001

Soner, B. C., Sahin, A. S., \& Sahin, T. K. (2013). A survey of Turkish hospital patients' uses of herbal medicine. European Journal of Integrative Medicine, 5(6), 547-552. https://doi.org/ 10.1016/j.eujim.2013.08.004

Toklu, H.Z. (2016). Pharmacovigilance of Herbal Medicine: Herbavigilance. Advances in Pharmacoepidemiology \& Drug Safety, 5(4). https://doi.org/10.4172/2167-1052.1000208

Tulunay, M., Aypak, C., Yikilkan, H., \& Gorpelioglu, S. (2015). Herbal medicine use among Turkish patients with chronic diseases. Journal of Intercultural Ethnopharmacology, 4(3), 217. https://doi.org/10.5455/jice.20150623090040

Zhang, Y., Peck, K., Spalding, M., Jones, B. G., \& Cook, R. L. (2012). Discrepancy between patient's use of and health providers' familiarity with CAM. Patient Education and Counselling, 89(3), 399-404. https://doi.org/10.1016/j.pec.2012.02.014 